\title{
Laparoscopic management of a giant hiatal hernia with gastric volvulus: a case report
}

\author{
Daniele Di Martino $^{1 \wedge}$, Mirko Barone $^{1 \wedge}$, Massimo Ippoliti $^{2}$, Felice Mucilli ${ }^{2,3} \wedge$ \\ ${ }^{1}$ Department of General and Oncological Surgery, SS. Annunziata Hospital, Chieti, Italy; ${ }^{2}$ Department of General and Thoracic Surgery, SS. \\ Annunziata Hospital, Chieti, Italy; ${ }^{3}$ University Department of Medical, Oral and Biotechnological Sciences, Gabriele d'Annunzio University of \\ Chieti-Pescara, Chieti, Italy \\ Correspondence to: Dr. Daniele Di Martino, MD. Department of General and Oncological Surgery, SS. Annunziata Hospital, Via dei Vestini n.1, \\ 66100, Chieti, Italy. Email: dr.danieledimartino@gmail.com.
}

\begin{abstract}
Giant hiatal hernias represent a small subset of diaphragmatic hernial disease. Characterized by supramesocolic or multidistrict intrathoracic evisceration, they are at greater risk of complications due to the tendency to strangulation and to meso- or organoaxial volvuli as a result of the end-stage of dysautonomic and dysmotilic processes involving the esophagogastric junction. Giant hiatal hernias fall into the category of well-being pathologies and are often concomitant with obesity. Since congenital aetiology is rare, the underlying pathophysiological mechanisms are increased intra-abdominal pressure, morbid obesity, Caucasian race. Nevertheless, this is usually an incidental finding in the absence of suggestive clinical findings and if symptoms arise, they are usually nonspecific (epigastralgia, bloating, reflux). On the other hand, the occurrence of hematemesis, epigastric or atypical chest pain represent worrisome symptoms that may underlie the onset of local complications such as incarceration and volvuli. Surgical management usually appears challenging, usually involving inveterate compartment defects as far as tissue loss of strength making repair complex and usually demanding mesh cruroplasties. A minimally invasive approach has a double utility: an accurate direct dissection control and the undeniable advantages of postoperative functional recovery. However, superiority of minimally invasive surgical approach still claims debate, especially concerning long-term outcomes. A clinical case of a patient with a giant hiatal hernia with organo-axial gastric volvulus and laparoscopic surgically repaired is reported.
\end{abstract}

Keywords: Hiatal hernia; laparoscopy; minimally invasive surgery; case report

Received: 10 December 2020; Accepted: 07 April 2021; Published: 30 June 2021.

doi: 10.21037/asj-20-4

View this article at: http://dx.doi.org/10.21037/asj-20-4

\section{Introduction}

Giant hiatal hernia is defined by the intrathoracic visceral encumbrance of at least $30 \%$ of the gastric volume, which is characterized by the simultaneous presence of typical gastrointestinal symptoms such as dysphagia, heartburn, regurgitation and atypical symptoms such as precordialgia, tachyarrhythmias, dyspnoea (1). Hence, we present a case of a 65 years-old female patient who addressed to our Department due to the onset of dysphagia and persistent emesis secondary to a complicated giant type III hiatal hernia with partial right side mesorgan-axial gastric volvulus. We present the following article in accordance with the CARE reporting checklist (available at https://asj.amegroups.com/ article/view/10.21037/asj-20-4/rc).

^ ORCID: Daniele Di Martino, 0000-0003-3042-1503; Mirko Barone, 0000-0002-3578-8657; Massimo Ippoliti, 0000-0003-19850542; Felice Mucilli, 0000-0003-1591-0325. 


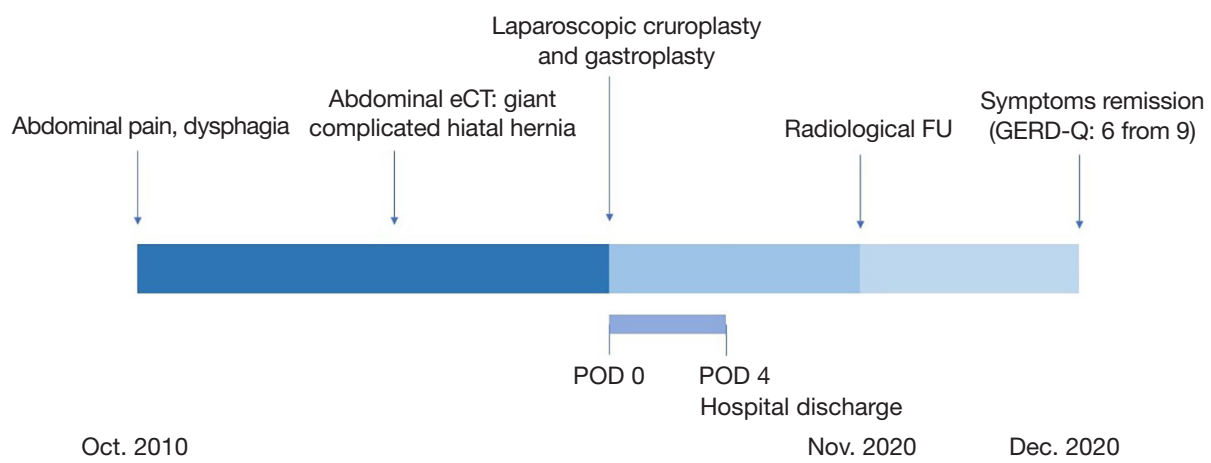

Figure 1 Timeline from onset of symptoms to postoperative follow-up.

\section{Case report}

A 65 years old female patients referred to our Department complaining abdominal pain, progressive dysphagia and short of breath (Figure 1). Her medical history was silent, except for mild morbidity obesity (BMI: $32.20 \mathrm{~kg} / \mathrm{m}^{2}$ ), hypertension and a hiatal hernia diagnosed twenty years ago. No previous abdominal surgery was reported. Physical examination revealed distension and upper quadrant tympanism without any sign of abdominal rebound or tenderness; while a mild leukocytosis (WBC: $\left.13 \times 10^{3} \mu \mathrm{L}\right)$ in the absence of significant increases in necrosis enzymes (LDH: $198 \mathrm{U} / \mathrm{L}$ ). The subsequent enhanced computed tomography revealed the presence of a giant hiatal hernia with gastric, omental and transverse colon content without visceral air-fluid levels with a consensual mesorgan-axial gastric volvulus on the gastroepiploic vessels and a S6-S8 right lower pulmonary lobe atelectasis (Figure 2). According to symptoms and undeniable risk of viscus strangulation, laparoscopic hiatal hernia repair was performed. A totally laparoscopic five port approach (four $12 \mathrm{~mm}$ sovraumbelical, bilateral pararectal and right flank and a $5 \mathrm{~mm}$ left ipocondrium ports for a Nathanson liver retractor) was scheduled. Abdominal exploration confirmed preoperative findings (Figure $3 A$ ). Procedure started with direct approach to the right diaphragmatic pillar in order to expose Juvara's fibers and the proper plane for gently dissection through the Leimer-Bertelli's membrane (Figure 3B). After peritoneal sac dissection from the hiatal surface area and the preparation of the retrogastric passage (Figure 3C), double split polylactic acid-based mesh diaphragmatic pillars cruroplasty through non-absorbable interrupted 2-0 sutures (Figure $3 D, E$ ) and a Nissen-Rossetti fundoplication was carried out (Figure $3 F$ ). Hospital stay was uneventful and oral intake was started on the second postoperative day. The patient was discharged on the fourth postoperative day. After On the first postoperative month, radiological follow-up revealed proper transit of the barium enema through the gastro-esophageal junction with regular findings of the stomach wrap (Figure $4 A, B$ ). The GERD-Q score at 1 and 2 months was 9 and 6 , respectively.

All procedures performed in this study were in accordance with the ethical standards of the institutional and national research committees and with the Helsinki Declaration (as revised in 2013). The publication of this manuscript is waived from patient informed consent according to the ethics committee or institutional review board.

\section{Discussion}

Giant hiatal hernia has an incidence ranging from $0.3 \%$ to $15 \%$ of all hiatal hernias (1). Progressively and constantly symptomatic, it represents the evolution of a loss of visceral domicile towards the middle mediastinum due to an acquired fibromuscular laxity of the phrenoesophageal membrane, the preaortic fascia and the median arcuate ligament (2). An impaired anatomical gastroesophageal junction (Bertelli's tear and diaphragmatic pillars laxity) as far as chronic inflammatory stimuli leading to esophageal muscular layers strictures constitute morphological predisposing factors. An intrathoracic visceral transposition could result either into organ- or into meso-axial herniation relating to gastric vascular system.

Cornerstones for proper surgical management of a giant hiatal hernia are an excellent visceral intra-abdominal dissection and reduction with a careful dissection, hernial sac care, performing an effective cruroplasty and the need for a tailored 

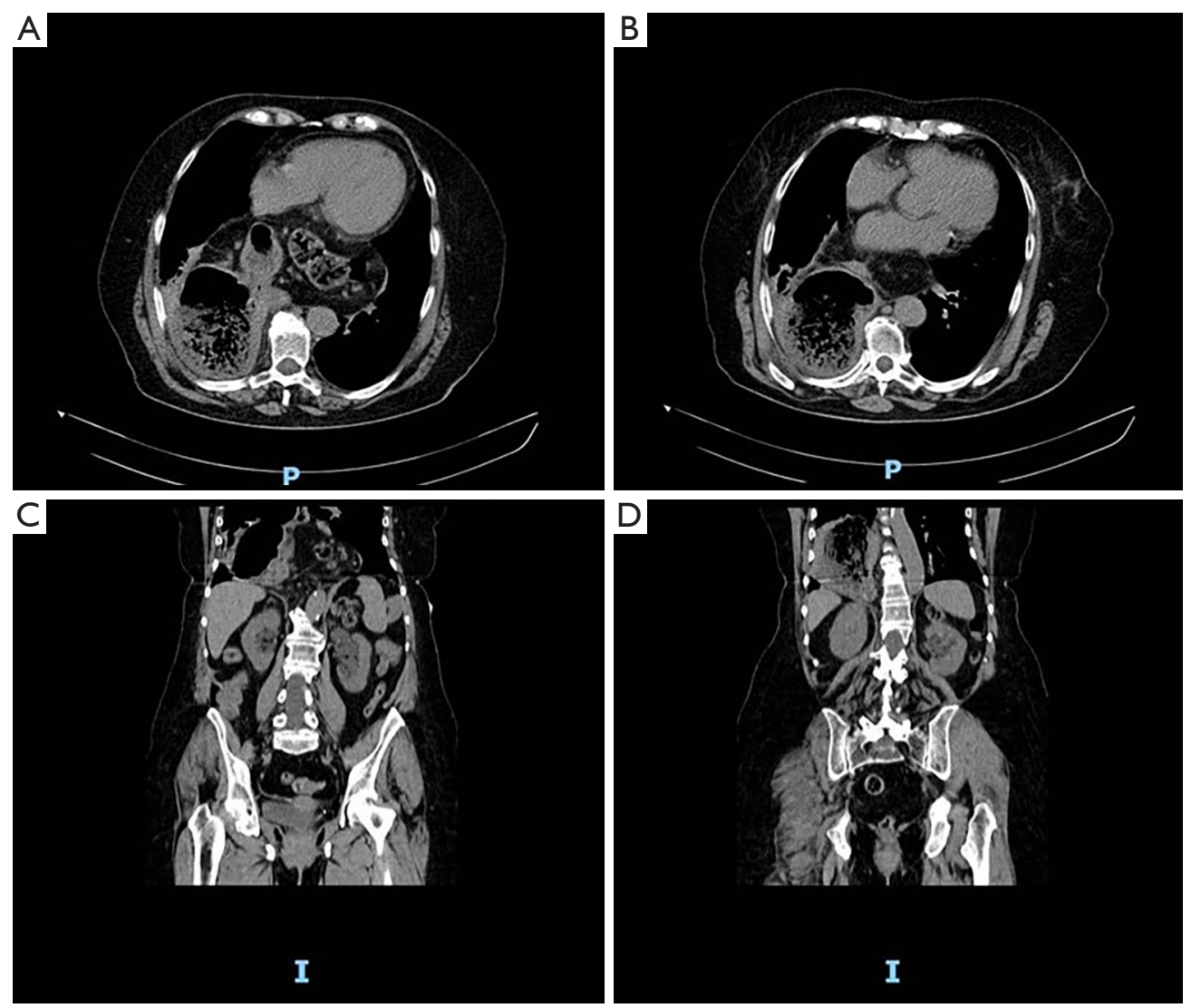

Figure 2 Preoperative abdominal CT: type III giant hiatal hernia with mesorgan-axial gastric volvulus.

gastropexy on the basis of patients' clinical and local conditions.

Intra-abdominal reduction of the hernia represents a key moment and allows the assessment of the extent of the para-hiatal area as well as a qualitative and quantitative evaluation of the anti-reflux mechanism defects. However, a giant hiatal hernia represents the end-stage of dysautonomic and dysmotilic processes in which an approach to anatomical dissection planes could be challenging. It is therefore essential to identify proper attachment plans. Specifically, hernial reduction must take place on embryonic attachment sheets (Jouvara's fibers in the right hemihiatus and Rougier's ones on the contralateral side) in order to correctly expose the posterior hiatal surface area delimited by the proximal third of the left diaphragmatic pillar laterally, by the tissue periaortic adipose cell posteriorly and by the posterior pericardium anteriorly. The procedure is completed by the resection of the mediastinal sac which allows a correct intra-abdominal descent of the esophagus and reduces the possibility of post-operative intramediastinal seromas.

Notwithstanding the undeniable advantages of minimally invasive approach to gastric volvulus in terms of faster postoperative recovery, a laparoscopic repair has been demonstrated both feasible and safe even if in debilitated patients according to a significative reduction of intraoperative and postoperative morbidities as far as a higher defined dissection planes. However, short and longterm outcomes comparing open and minimally invasive approach still claims debate due to equivalent results (3).

Mesh cruroplasty to repair of large esophageal hiatal hernia still claims debate. Furnée et al. (4), in a metaanalysis involving 1,264 unmatched patients (924 mesh vs. 340 suture cohorts), favoured allograft hiatoplasty with al overall recurrence incidence of $14.6 \%$ rather than $26.3 \%$ in the only suture group. Similarly, Sathasivam et al. (5), reported a pooled effect size for postoperative recurrence in favour of mesh repair (OR: $0.48, \mathrm{P}<0.05)$ without any differences in postoperative morbidity ( $\mathrm{OR}: 1.30, \mathrm{P}=0.36)$ resizing in some way the theoretical and, in any case, 

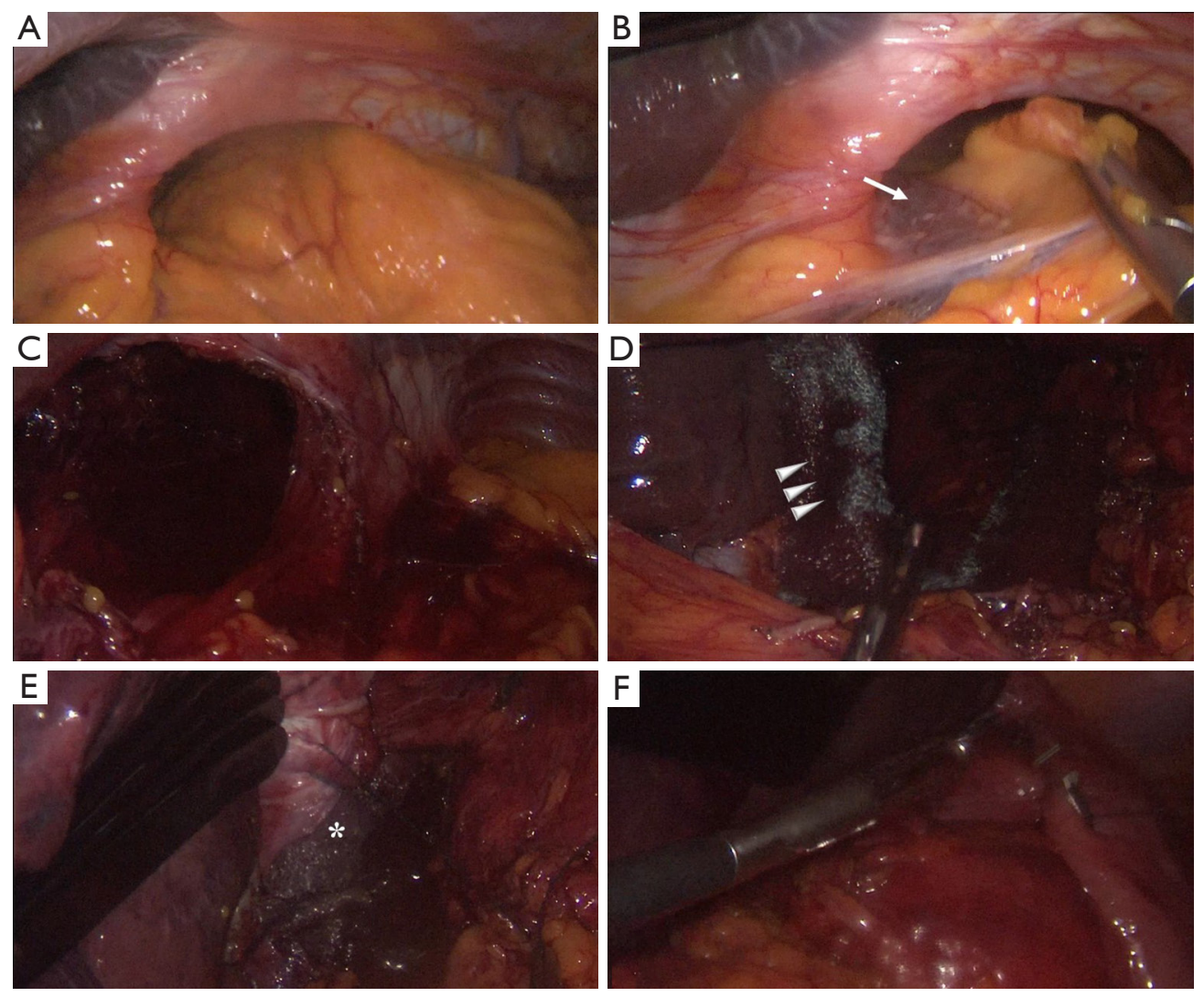

Figure 3 Intraoperative findings: (A) hiatal defect; (B) Juvara's fibers dissection; (C) diaphragmatic pillars and sac excision; (D,E) PLA-based cruroplasty; (F) Nissen-Rossetti gastric wrap.

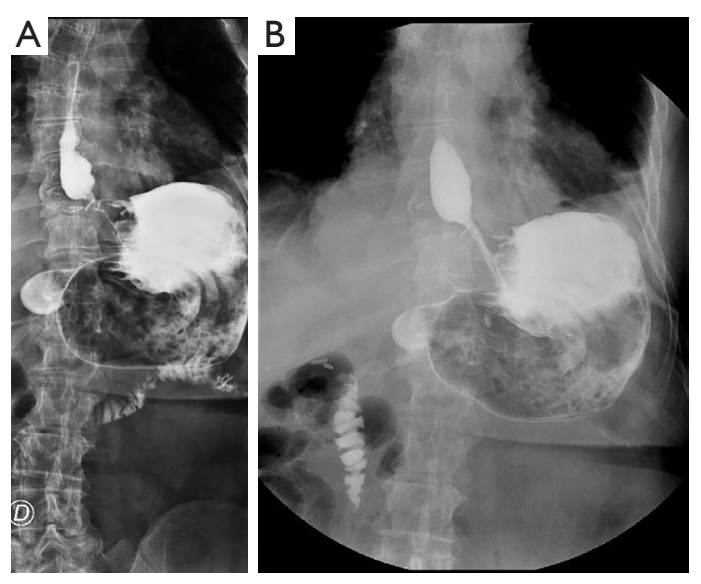

Figure 4 Postoperative barium enema.

described risk of post-operative erosions and decubitus which has significantly opposed its adoption in the past. Moreover, a prosthetic cruroplasty could reinforce the inert physiological muscular properties of hiatal fibers lacing of extracellular matrix which is a known risk factor for lack of postoperative plasticity as far as for both early and long-term recurrence (6).

Concerning with surgical approach and comparing laparoscopic and open surgery, several reports have demonstrated safety and efficacy of a minimally invasive strategy even if in complicated cases. However, universal and definitive evidences still lack. Proponents of a laparotomic treatment argue higher recurrence rates, as far as intraoperative complications. On the other hand, laparoscopy ensure better outcomes and 30-day mortality rates (odds reduction of 48\%); but, specific patients' circumstances suggesting a direct surgeons' intention to MIS are still scientifically unidentified and preferences still lay on surgical team skills and learning curves (7).

Finally, as regards with antireflux techniques, the extent of the hiatal defect, the theoretical risk of recurrence as well as the coexistence of concomitant functional esophagogastric symptoms cannot be disregarded. Evidence supporting an ideal strategy is 
still missing, with both partial and full wraps described. However, the main goal of anti-reflux plastic remains the preservation of correct post-operative anatomy and the reduction of post-operative reflux esophagitis, which is described up to $28 \%$ of patients with hiatal hernia. Trepanier et al. (8), in a retrospective study including 87 matched patients (Dor: $\mathrm{n}=48$ vs. Nissen: $\mathrm{n}=58$ ), showed a lower incidence of dysphagia in the Dor cohort (0vs. 13, 79\%, $\mathrm{P}=0.02)$ in the early postoperative period; but, in the mid-term follow-up, no difference between approaches were reported. Similarly, Su et al. (9) demonstrated no significant difference in GERD-Q scores between complete and partial wraps $(\mathrm{P}=0.207)$, as well as no differences in terms of operative times, intraoperative blood loss, hospitalization and postoperative complications.

In our case, gastroplasty was carried out according to Nissen-Rossetti due to the extent of the hiatal defect, the laxity of the pillars, the risk of a type II recurrence and the gap of the juxtacardial esophagus in which, a partial anchoring technique could not have avoided the persistence of chronic gastroesophageal reflux symptoms.

A minimally invasive abdominal approach in the treatment of chronic or complicated hiatal hernias should be preferred. Although previous evidence supported for a transthoracic approach, postoperative life-threatening respiratory complications in face of patients' average age at diagnosis or clinical symptoms must be kept in mind. Furthermore, a laparoscopic approach appears safe and allows an accurate anatomical dissection with both an optimal reduction and functional restoration of the esophagogastric junction. Upon the appearance of symptoms of an incipient hernial complication, primary surgical treatment is preferable to avoid ischemic or perforative episodes with subsequent high rates of perioperative morbidity and mortality.

\section{Acknowledgments}

Funding: None.

\section{Footnote}

Reporting Checklist: The authors have completed the CARE reporting checklist. Available at https://asj.amegroups.com/ article/view/10.21037/asj-20-4/rc

Conflicts of Interest: All authors have completed the ICMJE uniform disclosure form (available at https://asj.amegroups. com/article/view/10.21037/asj-20-4/coif). The authors have no conflicts of interest to declare.

Ethical Statement: The authors are accountable for all aspects of the work in ensuring that questions related to the accuracy or integrity of any part of the work are appropriately investigated and resolved. All procedures performed in the study were in accordance with the ethical standards of the institutional and national research committees and with the Helsinki Declaration (as revised in 2013). The publication of this manuscript is waived from patient informed consent according to the ethics committee or institutional review board.

Open Access Statement: This is an Open Access article distributed in accordance with the Creative Commons Attribution-NonCommercial-NoDeri 4.0 International License (CC BY-NC-ND 4.0), which permits the noncommercial replication and distribution of the article with the strict proviso that no changes or edits are made and the original work is properly cited (including links to both the formal publication through the relevant DOI and the license). See: https://creativecommons.org/ licenses/by-nc-nd/4.0/.

\section{References}

1. Mitiek MO, Andrade RS. Giant hiatal hernia. Ann Thorac Surg 2010;89:S2168-73.

2. Kahrilas PJ, Kim HC, Pandolfino JE. Approaches to the diagnosis and grading of hiatal hernia. Best Pract Res Clin Gastroenterol 2008;22:601-16.

3. Inaba K, Sakurai Y, Isogaki J, . Laparoscopic repair of hiatal hernia with mesenterioaxial volvulus of the stomach. World J Gastroenterol 2011;17:2054-7.

4. Furnée E, Hazebroek E. Mesh in laparoscopic large hiatal hernia repair: a systematic review of the literature. Surg Endosc 2013;27:3998-4008.

5. Sathasivam R, Bussa G, Viswanath Y, et al. 'Mesh hiatal hernioplasty' versus 'suture cruroplasty' in laparoscopic para-oesophageal hernia surgery; a systematic review and meta-analysis. Asian J Surg 2019;42:53-60.

6. Basso N, De Leo A, Genco A, et al. 360 degrees laparoscopic fundoplication with tension-free hiatoplasty in the treatment of symptomatic gastroesophageal reflux disease. Surg Endosc 2000;14:164-9.

7. Fullum TM, Oyetunji TA, Ortega G, et al. Open versus 
laparoscopic hiatal hernia repair. JSLS 2013;17:23-9.

8. Trepanier M, Dumitra T, Sorial R, et al.

Comparison of Dor and Nissen fundoplication after laparoscopic paraesophageal hernia repair. Surgery 2019;166:540-6.

doi: $10.21037 /$ asj-20-4

Cite this article as: Di Martino D, Barone M, Ippoliti M, Mucilli F. Laparoscopic management of a giant hiatal hernia with gastric volvulus: a case report. AME Surg J 202 1;1:9.
9. Su F, Zhang C, Ke L, et al. Efficacy comparison of laparoscopic Nissen, Toupet and Dor fundoplication in the treatment of hiatal hernia complicated with gastroesophageal reflux disease. Zhonghua Wei Chang Wai Ke Za Zhi 2016;19:1014-20. 